\title{
The poor accuracy of D-dimer
} for the diagnosis of prosthetic joint infection but its potential usefulness in early postoperative infections following revision arthroplasty for aseptic loosening

\author{
M. Fernandez-Sampedro ${ }^{*}$, I. Sanlés-González , C. García-lbarbia² , N. Fañanás-Rodríquez ${ }^{3}$,
} M. Fakkas-Fernández $z^{4}$ and M. C. Fariñas ${ }^{1}$

\begin{abstract}
Background: D-dimer was introduced in 2018 as an alternative biomarker for C-reactive protein (CRP) in the diagnostic of prosthetic joint infection (PJI) criteria of the Musculoskeletal Infection Society. We assessed the accuracy of plasma D-dimer for the diagnosis of early, delayed, and late PJI according to Infectious Diseases Society of America (IDSA) criteria, and whether persistently high levels of D-dimer in cases of aseptic loosening (AL) may be predictive of subsequent implant-related infection.

Methods: A prospective study of a consecutive series of 187 revision arthroplasties was performed at a single institution. Septic $(n=39)$ and aseptic revisions $(n=141)$ were classified based on IDSA criteria. Preoperative assessment of CRP, erythrocyte sedimentation rate (ESR) and D-dimer was performed. Receiver operating curves were used to determine maximum sensitivity and specificity of the biomarkers. The natural progress of D-dimer for AL cases was followed up either until the date of implant-related infection at any time during the first year or 1 year after revision in patients without failure. Clinical outcomes for those AL cases included infection-related failure that required a new surgery or need for antibiotic suppression.

Results: Preoperative D-dimer level was significantly higher in PJI cases than in AL cases $(p=0.000)$. The optimal threshold of D-dimer for the diagnosis of PJI was $1167 \mathrm{ng} / \mathrm{mL}$. For overall diagnosis of PJI, C-reactive protein (CRP) achieved the highest sensitivity (84.6\%), followed by erythrocyte sedimentation rate (ESR) and D-dimer (82\% and $71.8 \%$, respectively). Plasma D-dimer sensitivity was lower for all PJI types. When combinations of 2 tests were studied, the combined use of ESR and CRP achieved the best accuracy for all types of PJI (76.9\%). 4.25\% of AL cases had implant failure due to implant-related infection during the first year after the index revision arthroplasty, only the cases with early failure maintained high D-dimer levels.
\end{abstract}

\footnotetext{
*Correspondence: marta.fernandezs@scsalud.es

${ }^{1}$ Infectious Diseases Department, Hospital Universitario Marqués de

Valdecilla, University of Cantabria, IDIVAL, Av. Valdecilla s/n 39008,

Santander, Spain

Full list of author information is available at the end of the article
}

(c) The Author(s) 2022. Open Access This article is licensed under a Creative Commons Attribution 4.0 International License, which permits use, sharing, adaptation, distribution and reproduction in any medium or format, as long as you give appropriate credit to the original author(s) and the source, provide a link to the Creative Commons licence, and indicate if changes were made. The images or other third party material in this article are included in the article's Creative Commons licence, unless indicated otherwise in a credit line to the material. If material is not included in the article's Creative Commons licence and your intended use is not permitted by statutory regulation or exceeds the permitted use, you will need to obtain permission directly from the copyright holder. To view a copy of this licence, visit http://creativecommons.org/licenses/by/4.0/. The Creative Commons Public Domain Dedication waiver (http://creativeco mmons.org/publicdomain/zero/1.0/) applies to the data made available in this article, unless otherwise stated in a credit line to the data. 
Conclusions: Plasma D-dimer did not offer an improvement over the individual or combined diagnosis for any type of PJI according to IDSA criteria. Persistently raised levels of D-dimer after revision arthroplasty in AL cases might be used to effectively diagnose early postoperative infection.

Keywords: Plasma D-dimer, Prosthetic joint infection, PJl guidelines, First-line screening test

\section{Introduction}

In recent years, several scientific societies have developed criteria to standardize definitions for prosthetic joint infection (PJI) [1-4]. However, none have been widely adopted and the diagnostic approach to patients with suspected PJI remains extremely variable from center to center, depending on local experience, technological equipment, and adherence to available guidelines.

The main peripheral blood parameters used for the preoperative diagnosis of PJI include primarily 2 serological markers, serum C-reactive protein (CRP) and erythrocyte sedimentation rate (ESR). In the last 2 years, D-dimer has been recommended as a promising biomarker in suspected PJI, and has, in fact, been included in the 2018 Musculoskeletal Infection Society (MSIS) and International Consensus Meeting (ICM) criteria $[5,6]$. Relevant studies that used MSIS or ICM criteria addressing the evaluation of D-dimer for the diagnosis of PJI reach different conclusions [7-12]. This may be due to the different methodologies used among the studies and, because the thresholds of serological markers may vary, depending on the heterogeneity of the definitions, the time of infection, different D-dimer assays, and/or the infecting organism, therefore more work is needed to further validate their role in the diagnosis of PJI [6]

No published data are available on the role of D-dimer, taking into account the Infectious Diseases Society of America (IDSA) guidelines which describe the combined use of an abnormal ESR and CRP as the best combination for identifying patients with suspected PJI. In this study, we assessed the accuracy of plasma D-dimer in improving the preoperative diagnosis of early (less than 3 months after prosthesis implantation), delayed (between 3 and 12 months), and late PJI (>12 months after the prosthesis implantation) according to IDSA criteria, and whether the persistence of high levels of D-dimer in cases of aseptic loosening (AL) may be predictive of subsequent implant-associated infection.

\section{Materials and methods}

In this prospective observational study, all consecutive patients aged 18 years or older undergoing a total or partial revision of knee or hip arthroplasty were enrolled between February 2013 and February 2015. The study was conducted in the Division of Orthopedics of the
Hospital Universitario Marques of Valdecilla, a tertiary care hospital.

Patients were identified as having PJI according to IDSA guidelines [1]. Patients with a non-infectious diagnosis were defined as cases of AL: pain in the thigh or hip region, knee pain and radiological signs of loosening (inadequate initial fixation, mechanical loss of fixation over time, or biologic loss of fixation caused by particleinduced osteolysis around the implant). All patients with previous arthroplasty revisions were performed for noninfectious causes.

Since ESR, CRP and white blood cell count are part of our clinical routine; a preoperative determination was performed 2 weeks before the surgery. Based on receiveroperating-characteristic (ROC) analysis of the data, the cut-off level for CRP was $>1 \mathrm{mg} / \mathrm{dl}$, while for ESR it was $>15 \mathrm{~mm} / \mathrm{h}$. D-dimer levels were evaluated in heparin plasma samples using the Tina-quant D-Dimer immunoturbidimetric assay (Roche Diagnostics $\odot$ ) on the Cobas c501 analyzer (Roche $\odot$ ) [13].

The histopathological criteria established by Morawietz and Krem (Additional file 1: Appendix S1) [14] were used to define a standardized evaluation of the periprosthetic membrane. Venous blood samples were obtained preoperatively on the day of surgery, and D-dimer was determined in all study patients. Data (demographics, comorbidities, type of implant, surgical procedure, microbiological results of cultures and antimicrobial treatment) of all patients were collected prospectively by the clinical researchers of the institution using a standardized data collection form.

Patients with comorbidities that could increase plasma levels of D-dimer were excluded, e.g., arterial thromboembolic disease, myocardial infarction, stroke, acute limb ischemia, intracardiac thrombus within 4 weeks, venous thromboembolic disease, such as deep vein thrombosis, pulmonary embolism, disseminated intravascular coagulation, hypercoagulation disorder, periprosthetic fracture or joint dislocation within 2 weeks, active malignancy, infections in other regions of the body, skin ulcer or hematoma.

Patients with AL were followed up after inclusion in the study for a minimum of 24 months. Clinical outcomes include infection-related failure that required a new surgery or need for antibiotic suppression. The natural progress of D-dimer in non-PJI patients was followed 
up either until the date of implant-related infection at any time during the first year or 1 year after revision in patients without failure.

\section{Statistical analysis}

Statistical analysis was performed using Statistical Package for the Social Sciences Statistics for Windows version 25 (IBM Corporation, Armonk, NY, USA). Patient records were anonymized prior to analysis. Statistical analysis was performed by a physicist external to the recruitment and clinical management of the patients. Frequencies were given for categorical variables and compared in contingency tables using the Chi-squared test. Means were calculated for quantitative variables and compared using Student's t-test. A $p$-value of less than 0.05 was considered significant. The Youden index ( $J=$ sensitivity + specificity -1 ) based on its correspondence with the diagnostic of PJI was used to determine the optimal threshold value for D-dimer [15]. Based on the cut-off values, the sensitivity, specificity, positive predictive value (PPV), and negative predictive value (NPV) of serum markers were calculated. The combination of different diagnostic test was performed using an "AND" combination. The combination schemes were both tests positive (test 1 positive and test 2 positive, composite diagnostic positive).

\section{Ethics, registration, funding, and potential conflicts of interest}

The local ethics committee approved the study protocol (CTR2010.163) and all subjects provided oral informed consent before participation. This work was supported by the Fundación Marqués de Valdecilla, through the project API 11/9. IDIVAL, Instituto de Investigación
Valdecilla, Cantabria. Spain. The authors have no conflicts of interest to declare.

\section{Results}

\section{Study population}

A total of 187 cases in 185 patients were prospectively included during the study period. 7 cases $(3.8 \%)$ were excluded: 3 due to revision for dislocation, 2 due to a recent periprosthetic fracture, 1 due to a recent history of deep vein thrombosis, and 1 due to the presence of a venous leg ulcer. Of the remaining 180 cases, 141 (78.3\%) were diagnosed as $\mathrm{AL}$, while the other 39 (21.7\%) patients were defined as PJI. Of these, 7 (18\%) were classified as early, 9 (23\%) as delayed, and 23 (59\%) as late PJI. Detailed demographic data of all patients are presented in Table 1 . There were no statistically significant differences between the 2 groups (PJI and AL) in terms of age, gender, and comorbidities (mostly diabetes) $(p>0.05)$. Patients with PJI had a greater number of previous revisions than the non-PJI group $(p<0.013)$.

Preoperative plasma D-dimer level was significantly higher in PJI cases than in AL cases $(1968 \pm 1471 \mathrm{ng} /$ $\mathrm{mL}$ vs. $942 \pm 1085 \mathrm{ng} / \mathrm{mL} ; \mathrm{p}=0.000)$. Mean serum ESR and CRP values were also significantly higher among PJI patients; ESR was $39.16 \pm 28.40 \mathrm{~mm} / \mathrm{h}$ in the PJI group compared to $15.15 \pm 15.41 \mathrm{~mm} / \mathrm{h}$ in the $\mathrm{AL}$ group $(\mathrm{p}=0.000)$, while mean CRP was $3.47 \pm 3.72 \mathrm{mg} /$ $\mathrm{dL}$ in PJI cases compared with $0.78 \pm 1.57 \mathrm{mg} / \mathrm{dL}$ in AL cases $(p=0.000)$ (Table 1). The optimal threshold of D-dimer for the diagnosis of PJI was $1167 \mathrm{ng} / \mathrm{mL}$, and this value demonstrated a sensitivity of $72 \%$ and specificity of 75\% (75.3\%), AUC = 0.774 (0.693-0.854).

Table 1 Characteristics of study patients

\begin{tabular}{llll}
\hline Characteristics & AL $(\mathbf{n}=\mathbf{1 4 1})$ & PJ $(\mathbf{n}=\mathbf{3 9})$ & $\boldsymbol{P}$ value \\
\hline Age (year)-mean \pm SD & $69.23 \pm 11.14$ & $66.54 \pm 14.13$ & 0.220 \\
Male n (\%) & $60(42.6 \%)$ & $21(53.8 \%)$ & 0.123 \\
Body mass index-mean \pm SD & $30.58 \pm 5.2$ & $29.88 \pm 6.41$ & 0.542 \\
Hip n (\%) & $91(64.5 \%)$ & $27(69.2 \%)$ & 0.73 \\
Knee $n$ (\%) & $50(35.5 \%)$ & $12(30.8 \%)$ & 0.73 \\
Diabetes mellitus n (\%) & $25(17.7 \%)$ & $7(17.9 \%)$ & 0.86 \\
Immunosuppressive therapy n (\%) MTX & $2(2.1 \%)$ & $1(2.6 \%)$ & 0.62 \\
Systemic steroid therapy n (\%) & $2(1.4 \%)$ & 0 & 0.62 \\
Prior revision arthroplasty n (\%) & $38(27 \%)$ & $20(51.3 \%)$ & 0.011 \\
CRP mean \pm SD & $0.78 \pm 1.57$ & $3.47 \pm 3.72$ & 0.00011 \\
ESR mean \pm SD & $15.15 \pm 15.41$ & $39.16 \pm 28.40$ & 0.000013 \\
D-dimer mean \pm SD & $942.36 \pm 1085.78$ & $1968.54 \pm 1471.84$ & 0.00025 \\
\hline
\end{tabular}

PJI, Prosthetic joint infection; AL, Aseptic loosening; MTX, Methotrexate; CRP, C-reactive protein; ESR, erythrocyte sedimentation rate; SD, Standard Deviation 


\section{Comparison of individual diagnostic parameters for the diagnosis of PJI}

According to individual parameters for the overall diagnosis of PJI, CRP achieved the highest sensitivity (84.6\%), followed by ESR and D-dimer (82\% and $71.8 \%$, respectively). In early PJI diagnosis, ESR showed the highest sensibility (100\%) followed by both CRP and D-dimer (85.7\%). For delayed PJI, both CRP and ESR had the highest sensibility $(88.8 \%)$, followed by D-dimer $(66.6 \%)$ and, in late PJI, the highest sensibility was obtained with CRP (82.6\%), followed by D-dimer and ESR (73.9\% and 69.5\%, respectively) (Table 2 ).

\section{Comparison of grouped diagnostic parameters for the diagnosis of $\mathrm{PJI}$}

When evaluating potential combinations of 2 tests in the detection of PJI, we found that the overall combined sensitivity and specificity for ESR + CRP was $76.9 \%$ and $87.8 \%$, respectively, $59 \%$ and $92.7 \%$ for CRP + D-dimer, and $61.5 \%$ and $88.3 \%$ for ESR + D-dimer. For each type of infection, ESR + CRP diagnosed $85.7 \%$ of early, $77.7 \%$ of delayed, and $73.9 \%$ of late cases. The combination of CRP + D-dimer diagnosed $57.1 \%$ of early, $44.4 \%$ of delayed, and $65.2 \%$ of late cases. Finally, the addition of ESR + D-dimer diagnosed $71.4 \%$ of early, $44.4 \%$ of delayed and $65.2 \%$ of late cases (Table 3). Additional file 2: Appendix S2 shows the diagnostic accuracy of individual and grouped preoperative tests in PJI.

\section{Outcome}

$6(4.25 \%)$ of the $141 \mathrm{AL}$ cases in our cohort had implant failure due to implant-related infection. All failures occurred during the first year after the index revision arthroplasty and, although all of them showed high levels of circulating D-dimer at the time of diagnosis, only 2 cases of early failure ( $<3$ months) maintained high levels of D-dimer. In both, virulent microorganisms, Staphylococcus aureus and Enterobacter cloacae, were isolated, and these patients showed raised levels of ESR and CRP after postoperative day 1 that peaked at postoperative day 4 , and then remained elevated until the time of the failure, whereas plasma D-dimer showed persistently high levels that peaked at the time of the implant failure (Table 4).

\section{Microbiology and serological markers}

43 microorganisms were isolated from 39 PJI patients. Only 1 patient was culture-negative and a respiratory source of infection was identified. Serological markers (ESR, CRP and D-dimer) based on the infecting organisms isolated were then evaluated (Table 5).

\section{Discussion}

To our knowledge, this is the first study that evaluates D-dimer levels in the diagnosis of PJI according to IDSA guidelines which highlights the use of both an abnormal ESR and CRP as the best combination for patients with suspected PJI. Our results show that plasma D-dimer did not improve the individual diagnosis of ESR or CRP for any type of PJI. The sensitivity of plasma D-dimer was lower for all PJI types, except for early infection in which it offered the second-best sensitivity, equal to ESR. When combinations of 2 tests were studied, the combined use of ESR and CRP achieved the best accuracy for all types

Table 2 Diagnostic Accuracy of individual Preoperative Tests according to type of PJI

\begin{tabular}{|c|c|c|c|c|c|}
\hline Markers & Specificity (\%) & Sensitivity (\%) & PPV (\%) & NPV (\%) & $\operatorname{AUC}(95 \% \mathrm{Cl})$ \\
\hline \multicolumn{6}{|c|}{ D-dimer $>1167$ ng/mL } \\
\hline All PJI & $75.3(69-83)$ & $71.8(58-86)$ & $45.9(33.3-58.4)$ & $90.4(81.1-99.7)$ & $0.774(0.693-0.854)$ \\
\hline Early & $75.3(69-83)$ & $85.7(60-100)$ & $15.4(4-26.7)$ & $99(88-100)$ & $0.863(0.785-0.942)$ \\
\hline Delayed & $75.3(69-83)$ & $66.6(36-97)$ & $15(4-26.7)$ & $97.1(86.8-100)$ & $0.721(0.555-0.887)$ \\
\hline Late & $75.3(69-83)$ & $69.5(51-88)$ & 32.7 (19.5-46.8) & 93.7 (83.9-1009 & $0.767(0.665-0.87)$ \\
\hline \multicolumn{6}{|c|}{$\mathrm{CRP}>1 \mathrm{mg} / \mathrm{dL}$} \\
\hline All PJI & $82.1(76-88)$ & $84.6(73-96)$ & $56.8(44-69)$ & $95(87-100)$ & $0.869(0.797-0.940)$ \\
\hline Early & $82.1(76-88)$ & $85.7(60-100)$ & $19.3(5-33)$ & $99.13(91-100)$ & $0.828(0.612-1)$ \\
\hline Delayed & $82.1(76-88)$ & $88.8(68-100)$ & $24.2(9.6-38.8)$ & $99.1(91-100)$ & $0.936(0.885-0.986)$ \\
\hline Late & $82.1(76-88)$ & $82.6(67-98)$ & $43.2(28.5-57.8)$ & $96.6(88.5-100)$ & $0.863(0.772-0.953)$ \\
\hline \multicolumn{6}{|c|}{$\mathrm{ESR}>15 \mathrm{~mm} / \mathrm{h}$} \\
\hline All PJI & $72.8(65-80)$ & $82(70-94)$ & $45.7(34-57.4)$ & $93.5(82.8-100)$ & $0.792(0.7-0.885)$ \\
\hline Early & $72.8(65-80)$ & $100(100-100)$ & $15.5(5-26)$ & $100(88-100)$ & $0.897(0.828-0.967)$ \\
\hline Delayed & $72.8(65-80)$ & $88.8(63-100)$ & $17.3(6.4-28.3)$ & $99(87.3-100)$ & $0.866(0.765-0.968)$ \\
\hline Late & $72.8(65-80)$ & $73.9(56-92)$ & $30.9(18.7-43.1)$ & $94.4(18.7-43.1)$ & $0.767(0.592-0.871)$ \\
\hline
\end{tabular}

PJI, Prosthetic joint infection; PPV, Positive predictive value; NPV, Negative predictive value; AUC, Area under the curve; $\mathrm{Cl}$, Confidence interval 
Table 3 Diagnostic Accuracy of Combined Preoperative Tests according to type of PJI

\begin{tabular}{|c|c|c|c|c|c|}
\hline Markers & Specificity (\%) (95\% CI) & Sensitivity (\%) (95\% Cl) & PPV (\%) $(95 \% \mathrm{Cl})$ & NPV (\%) $(95 \%$ Cl) & AUC $(95 \% \mathrm{Cl})$ \\
\hline \multicolumn{6}{|l|}{ D-dimer + CRP } \\
\hline All PJl patients & $92.7(88.3-97)$ & $59(43.5-74.4)$ & $69.7(54-85.4)$ & $88.8(83.6-94)$ & $0.885(0.831-0.938)$ \\
\hline Early & $92.7(88.3-97)$ & $57.1(20.5-93.8)$ & $28.6(4.9-52.2)$ & $97.7(93-100)$ & $0.944(0.902-0.985)$ \\
\hline Delayed & $92.7(88.3-97)$ & $44.4(12-76.9)$ & $28.6(5-52.2)$ & $96.2(91.6-100)$ & $0.914(0.853-0.975)$ \\
\hline Late & $92.7(88.3-97)$ & $65.2(45.7-84.7)$ & $60(40.8-79.2)$ & $94.1(89.6-98.5)$ & $0.855(0.782-0.928)$ \\
\hline \multicolumn{6}{|l|}{ D-dimer + ESR } \\
\hline All PJl patients & $88.3(82.9-93.7)$ & $61.5(46.3-76.8)$ & $60(44.8-75.2)$ & $89(83.5-94.4)$ & $0.812(0.729-0.896)$ \\
\hline Early & $88.3(82.9-93.7)$ & $71.4(38-100)$ & $23.8(5.6-42)$ & $98.4(92-100)$ & $0.917(0.856-0.978)$ \\
\hline Delayed & $88.3(82.9-93.7)$ & $44.4(12-76.9)$ & $20(2.4-37.5)$ & $96(89.9-100)$ & $0.872(0.786-0.957)$ \\
\hline Late & $88.3(82.9-93.7)$ & $65.2(45.7-84.7)$ & $48.4(30.8-66)$ & $93.8(87.9-99.7)$ & $0.7358(0.633-0.882)$ \\
\hline \multicolumn{6}{|l|}{$C R P+E S R$} \\
\hline All PJl patients & $87.8(82.4-93.3)$ & $76.9(63.7-90.1)$ & $63.8(50-77.6)$ & $93.2(87.3-99)$ & $0.838(0.757-0.919)$ \\
\hline Early & $87.8(82.4-93.3)$ & $85.7(59.8-100)$ & $26(8.1-44)$ & $99,1(92.77-100)$ & $0.903(0.83-0.976)$ \\
\hline Delayed & $87.8(82.4-93.3)$ & $77.7(50.6-100)$ & $29.2(11-47.3)$ & $98.4(92-100)$ & $0.926(0.869-0.984)$ \\
\hline Late & $87.8(82.4-93.3)$ & $73.9(56-91.8)$ & $50(33.2-66.8)$ & $95.3(89.2-100)$ & $0.783(0.66-0.919)$ \\
\hline
\end{tabular}

PJI, Prosthetic joint infection; PPV, Positive predictive value; NPV, Negative predictive value; AUC, Area under the curve; $\mathrm{Cl}$, Confidence interval

Table 4 Aseptic loosening patients with failure due to implant-related infection

\begin{tabular}{|c|c|c|c|c|c|c|c|c|}
\hline \multirow[b]{2}{*}{ Case } & \multicolumn{4}{|c|}{ Index revision surgery } & \multicolumn{4}{|c|}{ Following revision due to infection } \\
\hline & $\mathrm{ESR} \mathrm{mm} / \mathrm{h}$ & CRP mg/dl & D-dimer $\mathrm{ng} / \mathrm{mL}$ & $\begin{array}{l}\text { Time to } \\
\text { failure } \\
\text { (months) }\end{array}$ & $\mathrm{ESR} \mathrm{mm} / \mathrm{h}$ & CRP mg/dl & D-dimer $\mathrm{ng} / \mathrm{mL}$ & Microbiology \\
\hline$\# 1$ & 14 & 0.5 & 212 & 10 & ND & ND & 1323 & Staphylococcus epidermidis \\
\hline$\# 2$ & 5 & 0.2 & 118 & 1 & 22 & 2.1 & 2583 & Methicillin-sensitive S. aureus \\
\hline \#3 & 4 & 0.5 & 768 & 11 & 7 & 1 & 1529 & Staphylococcus epidermidis \\
\hline \#4 & 8 & 0.2 & 840 & 4 & 74 & 0.4 & 2782 & Staphylococcus epidermidis \\
\hline \#5 & 10 & 0.4 & 670 & 2 & 35 & 15.3 & 2498 & Enterobacter cloacae \\
\hline$\# 6$ & 10 & 0.1 & 476 & 10 & 8 & 0.1 & 1331 & Coagulase-negative staphylococci \\
\hline
\end{tabular}

Values in bold are elevated values within the reference range for each parameter

CRP, C-reactive protein; ESR, erythrocyte sedimentation rate; ND, Not done

of PJI, and the addition of D-dimer to ESR achieved the second-best sensitivity for early PJI.

In the last 2 years, D-dimer has been recommended as a promising biomarker in suspected PJI. Indeed, D-dimer was introduced in 2018 as an alternative biomarker with a cut-off value of $860 \mathrm{ng} / \mathrm{mL}$ in the new MSIS and ICM validated diagnostic PJI criteria [5, 6]. Recently, 8 metaanalyses addressing the diagnostic accuracy of D-dimer in PJI highlighted the wide heterogeneity among studies, because of that, univariate meta-regression and subgroup analysis were performed and found that the type of sample (serum or plasma) could be the primary factor affecting the heterogeneity of sensitivity, while the country of origin of the study (China and USA) may be the main source of heterogeneity in terms of specificity [16-23].

Seven studies have investigated plasma D-dimer in the diagnosis of PJI, including 6 Asian studies and 1
European study (Germany) (Table 6) [24-30]. On the basis of our study conducted according to IDSA guidelines, plasma D-dimer achieved the second-best sensitivity and the third-best specificity when compared with the aforementioned studies. Our results are in close agreement with those published in Asian populations, in terms of both sensitivity and specificity, and contrast with the European study. In this regard, 2 studies on serum D-dimer that recruited their patients from an American population using MSIS criteria and a threshold value of $850 \mathrm{ng} / \mathrm{mL}$ showed conflicting results, with sensitivities and specificities of $89 \%$ and $93 \%$ vs $96 \%$ and $32 \%$, respectively [7-10]. Therefore, it should be reevaluated whether racial differences are a major factor affecting the outcome of the different studies.

Among other concerns, the optimal cut-off value for D-dimer and its sensitivity and specificity remain 
Table 5 Values of ESR, CRP and D-dimer according to microorganisms in predicting infection

\begin{tabular}{|c|c|c|c|}
\hline Microorganisms & $\operatorname{ESR}(>15 \mathrm{~mm} / \mathrm{h})$ & $\mathrm{CRP}(>1 \mathrm{mg} / \mathrm{dl})$ & $\begin{array}{l}\text { D-dimer } \\
(>1167 \mathrm{ng} / \\
\mathrm{mL})\end{array}$ \\
\hline S. epidermidis $(n=18)$ & $14 / 18$ & $15 / 18$ & $16 / 18$ \\
\hline Early & $2 / 2$ & $2 / 2$ & $2 / 2$ \\
\hline Delayed & $2 / 3$ & $3 / 3$ & $3 / 3$ \\
\hline Late & $10 / 13$ & $10 / 13$ & $11 / 13$ \\
\hline Other CoNS $(n=11)^{a}$ & $9 / 11$ & $9 / 11$ & $4 / 11$ \\
\hline Early & $2 / 2$ & $2 / 2$ & $2 / 2$ \\
\hline Delayed & $4 / 4$ & $4 / 4$ & $1 / 4$ \\
\hline Late & $3 / 5$ & $3 / 5$ & $1 / 5$ \\
\hline Streptococcus spp. $(n=3)$ & $3 / 3$ & $3 / 3$ & $3 / 3$ \\
\hline Early & 0 & 0 & 0 \\
\hline Delayed & $1 / 1$ & $1 / 1$ & $1 / 1$ \\
\hline Late & $2 / 2$ & $2 / 2$ & $2 / 2$ \\
\hline Corynebacterium spp. $(n=3)$ & $3 / 3$ & $3 / 3$ & $3 / 3$ \\
\hline Early & $1 / 1$ & $1 / 1$ & $1 / 1$ \\
\hline Delayed & 0 & 0 & 0 \\
\hline Late & $2 / 2$ & $2 / 2$ & $2 / 2$ \\
\hline Gram-negative $(n=4)$ & $3 / 4$ & $3 / 4$ & $3 / 4$ \\
\hline Early & $3 / 3$ & $2 / 3$ & $3 / 3$ \\
\hline Delayed & 0 & 0 & 0 \\
\hline Late & $0 / 1$ & $1 / 1$ & $0 / 1$ \\
\hline Others $(n=4)^{b}$ & $3 / 4$ & $4 / 4$ & $2 / 4$ \\
\hline Early & $1 / 1$ & $1 / 1$ & $1 / 1$ \\
\hline Delayed & $2 / 2$ & $2 / 2$ & $1 / 2$ \\
\hline Late & $0 / 1$ & $1 / 1$ & $0 / 1$ \\
\hline
\end{tabular}

ESR, Erythrocyte sedimentation rate; CRP, C-reactive protein

a Other CoNS (coagulase-negative Staphylococci): Staphylococcus lugdunensis $(n=4)$, Staphylococcus capitis $(n=3)$, Staphylococcus caprae $(n=2)$, Staphylococcus haemolyticus $(\mathrm{n}=1)$, Staphylococcus hominis $(\mathrm{n}=1)$

${ }^{b}$ Others: Enterococcus faecalis ( $\left.n=2\right)$, Peptostreptococcus asaccharolyticus $(n=1)$, methicillin-sensitive Staphylococcus aureus $(n=1)$

unclear. Some authors considered that patients' body mass index (BMI), age or sex may comprise a confounding factor resulting in higher levels of $\mathrm{D}$-dimer, while women and older patients may have higher serum levels of D-dimer when used in the diagnosis of thrombosis and/or embolism [31]. In our cohort, the threshold for plasma D-dimer was similar to that described in the Asian population, particularly by Huang et al. [29], who also reported lower mean BMIs and lower median age than the Europeans. Recently, Grzelecki et al., found that the influence of the operated joint type may be a very important factor in the concentration of $\mathrm{D}$-dimer and that plasma $\mathrm{D}$-dimer has a relatively high value in the detection of knee PJI, but a moderate to low value for hip PJI [32]. It does seem logical that these characteristics could have unbalanced the optimal threshold of D-dimer for the diagnosis of PJI which ranged between $410 \mathrm{ng} / \mathrm{mL}$ to $2300 \mathrm{ng} / \mathrm{mL}$. In short, pending more robust studies that provide new data and investigate their correlations with sample type, comorbidities, ethnic variability, joint type, PJ type, etc., each institution should optimize D-dimer thresholds.

A further point to be discussed is whether plasma or serum is the most appropriate sample for performing D-dimer testing. The coagulant factor present in plasma but not in serum interferes with many biochemical parameters and the higher metabolite concentrations in serum offer more sensitive results in biomarker detection. Korte et al. demonstrated a close agreement of D-dimer concentrations determined from citrated plasma samples and serum for the exclusion of venous thromboembolism [33]. However, they added that the use of serum for D-dimer determination needs to be evaluated and validated in a clinical study. A recent meta-analysis of prosthesis-related infections showed that serum D-dimer outperformed plasma D-dimer in sensitivity and 


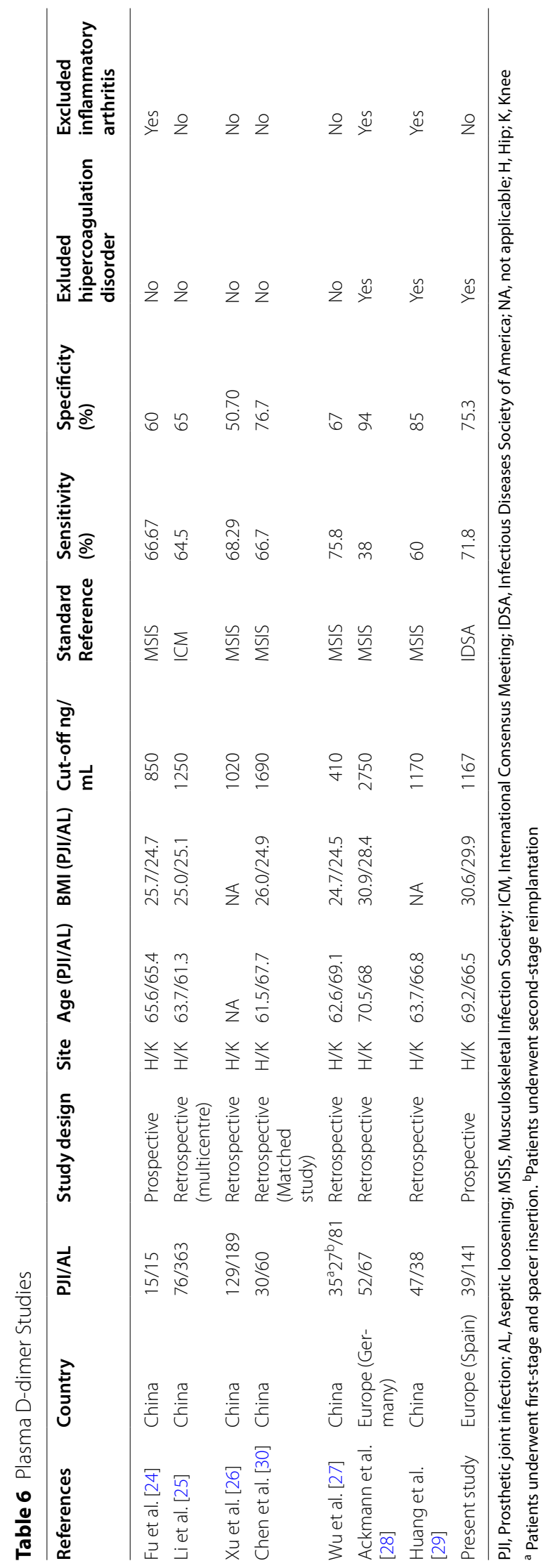


specificity ( 0.86 and 0.84 vs. 0.67 and 0.60 , respectively) [34]. In this regard, 6 studies on serum D-dimer in PJI were published between 2017 and 2020, 2 from the USA and 4 from China. Five used MSIS criteria for the diagnosis of PJI and the other used ICM. The D-dimer threshold ranged from 850 to $1170 \mathrm{ng} / \mathrm{mL}$ [7-12]. Unfortunately, they reached different conclusions and these results raise the question of whether differences in the type of sample are a major factor affecting the substantial discrepancies between studies.

In a prospective study that measured D-dimer levels before and after primary total hip or knee arthroplasty, the most significant changes in D-dimer levels were observed during the early postoperative period. Levels increased sharply and peaked on the first day after joint replacement surgery, decreasing to baseline levels on the following day [35]. In our AL cohort, $4.25 \%$ of the cases had implant failure due to infection. All of them had high levels of circulating D-dimer at the time of diagnosis. However, in the 2 cases with early failure, D-dimer levels remained raised and peaked at the time of failure. In these 2 cases, ESR and CRP levels peaked before the time failure was diagnosed. It would be of interest to study a larger number of cases with early failure to determine if there is any difference.

Finally, coagulase-negative staphylococci (CoNS) (67.4\%) were the most common pathogens isolated in our study, while S. epidermidis was the most prevalent species, particularly in late infections. Higher plasma D-dimer levels were associated with a slight tendency towards the detection of more S. epidermidis isolates than other inflammatory markers, but in contrast were associated with fewer PJI infections due to other CoNS isolates. We are unaware if small colony variants or biofilm formation by different CoNS play a role in these findings. No differences were found for other isolates such as low-virulence bacteria.

Our study has several limitations that should be considered. First, this is a single-center prospective study with potential for uncontrolled selection biases; however, methodologies were applied in a standard fashion, something that does not occur when patients are included from multiple centers where the test result of D-dimer can be interpretated as positive or negative with different thresholds and, therefore any change in thresholds may have a substantial impact on its diagnostic value. Second, the sample size for PJI infections in the study was small due to the low incidence of PJI and may lack statistical power to detect some associations in different subtypes of PJI being this limitation is described in other studies [30]. Third, there is no gold standard for the diagnosis of PJI and it is possible that the lack the sensitivity in detecting PJI, specially in chronic or low-grade infections. Fourth, we excluded patients with conditions that may induce high expression of D-dimer and could result in high false positive rates. These pathologies accounted for almost $4 \%$ of the study patients and, this low prevalence, does not appear to have been a bias for the study. However, different D-dimer assays may point to differences in sensitivity and specificity between studies. This emphasizes the need for standardization of D-dimer assays. Fifth, prior revisions of PJI group differed significantly from AL group which may affect the validity of the results. It has been recognized that the risk of PJI increases with the number of previous joint arthroplasties. However, it has been unclear whether the increased risk of PJI in patients with prior joint arthroplasties is due to an increased number of comorbid conditions, a prolonged operating time, an increased number of blood transfusions, or higher frequency of postoperative wound complications. Therefore, the identification of patients at high risk for PJI would allow for improved preoperative risk assessment, increase the index suspicion, and identify patients for whom focused efforts at prevention are necessary.

In conclusion, plasma D-dimer determined according to IDSA guidelines did not improve the individual or combined diagnosis of ESR or CRP for any type of PJI. The persistence of raised levels of plasma D-dimer after revision arthroplasty in AL cases might be used effectively in diagnosing early postoperative infection. Because of the remaining concerns surrounding this new serological biomarker in implant-associated infection, we urge caution in accepting serum and/or plasma D-dimer as a first-line screening test for PJI diagnosis, regardless of the guidelines adopted. This conclusion should be confirmed in appropriate clinical trials.

\section{Abbreviations}

AL: Aseptic loosening; AUC: Area under the Curve; BMI: Body mass index: CoNS: Coagulase-negative staphylococci; CRP: C-reactive protein; ESR: Erythrocyte sedimentation rate; ICM: International Consensus Meeting; IDSA: Infectious Diseases Society of America; MSIS: Musculoskeletal Infection Society; NPV: Negative predictive value; PJI: Prosthetic Joint Infection; PPV: Positive predictive value; ROC: Receiver operating characteristic.

\section{Supplementary Information}

The online version contains supplementary material available at https://doi. org/10.1186/s12879-022-07060-8.

Additional file 1: Appendix S1. Histopathological classification of the periprosthetic membrane.

Additional file 2: Appendix S2. Diagnostic Accuracy of individual preoperative tests and combinated test in PJI. PPV Positive predictive value; NPV Negative predictive value; AUC Area under the curve; Cl Confidence interval. 


\section{Acknowledgements}

The authors are grateful to the participating patients for their collaboration and to the Fundación Marqués de Valdecilla.

\section{Authors' contributions}

Conceived and designed study: MFS, MCF. Collection of data MFS, NFR, ISG, MFF. Analyze the data: MFS, CGI. Manuscript preparation: MFS, MCF. All authors read and approved the final manuscript.

\section{Funding}

This work was supported by the Fundación Marqués de Valdecilla, through the project API 11/9. IDIVAL, Instituto de Investigación Valdecilla, Cantabria, Spain.

\section{Availability of data and materials}

The database used and/or analysed during the current study are available from the corresponding author on reasonable request.

\section{Declarations}

\section{Ethics approval and consent to participate}

The study was performed in accordance with the Declaration of Helsinki. The protocol was reviewed and approved by the Clinical Research Ethics Committee of Cantabria (CTR2010.163) according to local standards.

\section{Consent for publication}

Not applicable.

\section{Competing interests}

The authors declare that they have no competing interests.

\begin{abstract}
Author details
${ }^{1}$ Infectious Diseases Department, Hospital Universitario Marqués de Valdecilla, University of Cantabria, IDIVAL, Av. Valdecilla s/n 39008, Santander, Spain. ${ }^{2}$ Internal Medicine Department, Hospital Universitario Marqués de Valdecilla, University of Cantabria, Santander, Spain. ${ }^{3}$ Clinical Analysis Department, Hospital Universitario Marqués de Valdecilla, Santander, Spain. ${ }^{4}$ Orthopaedic Surgery Department, Hospital Universitario Marqués de Valdecilla, Santander, Spain.
\end{abstract}

Received: 11 September 2021 Accepted: 13 January 2022

Published online: 27 January 2022

\section{References}

1. Osmon DR, Berbari EF, Berendt AR, Lew D, Zimmerli W, Steckelberg JM, Rao N, Hanssen A, Wilson WR. Diagnosis and management of prosthetic joint infection: clinical practice guidelines by the Infectious Diseases Society of America. Clin Infect Dis. 2013;56(1):e1-25.

2. Parvizi J, Zmistowski B, Berbari EF, Bauer TW, Springer BD, DellaValle CJ, Garvin KL, Mont MA, Wongworawat MD, Zalavras CG. New definition for periprosthetic joint infection: from the workgroup of the Musculoskeletal infection Society. Clin Orthop Relat Res. 2011;469:2992e4.

3. Parvizi J, Gehrke T, Chen AF. Philadelphia 2013: international consensus meeting on periprosthetic joint infection. Bone Joint J. 2013;95-B(11):1450-2

4. McNally M, Sousa R, Wouthuyzen-Bakker M, Chen AF, Soriano A, Vogely HC, Clauss M, Higuera CA, Trebše R. The EBJIS definition of periprosthetic joint infection: a practical guide for clinicians. Bone Joint J. 2021;103B(1):18-25 (Published online 2021 Jan 1).

5. Parvizi J, Tan TL, Goswami K, Higuera C, Della Valle C, Chen AF, Shohat $N$. The 2018 definition of periprosthetic hip and knee infection: an evidence-based and validated criteria. J Arthroplasty. 2018;33:1309e1314. e2.

6. Shohat N, Bauer T, Buttaro M, Budhiparama N, Cashman J, Della Valle CJ, et al. Hip and Knee Section, What is the definition of a Periprosthetic Joint Infection (PJI) of the knee and the hip? Can the same criteria be used for both joints?: Proceedings of International Consensus on Orthopedic Infections. J Arthroplasty. 2019; 34(2):S325-S7.

7. Shahi A, Kheir MM, Tarabichi M, Hosseinzadeh HRS, Tan TL, Parvizi J. Serum D-dimer test is promising for the diagnosis of periprosthetic joint infection and timing of reimplantation. J Bone Joint Surg Am. 2017;99:1419-27.

8. Huang J, Zhang Y, Wang Z, Dong Y, Zhao Y, Zheng J, Lian H, Jin Y. The serum level of D-dimer is not suitable for distinguishing between prosthetic joint infection and aseptic loosening. J Orthop Surg Res. 2019;14(1):407

9. Xiong L, Li S, Dai M. Comparison of D-dimer with CRP and ESR for diagnosis of periprosthetic joint infection. J Orthop Surg Res. 2019;14(1):240

10. Pannu TS, Villa JM, Patel PD, Riesgo AM, Barsoum WK, Higuera CA. The utility of d-dimer for the diagnosis of periprosthetic joint infection in revision total hip and knee arthroplasty. J Arthroplast. 2020;35:1692-5.

11. Qin L, Li F, Gong X, Wang J, Huang W, Hu N. Combined measurement of D-dimer and c-reactive protein levels: Highly accurate for diagnosing chronic periprothetic joint infection. J Arthroplast. 2020;35:229-34.

12. Hu Q, Fu Y, Tang L. Serum D-dimer as a diagnostic index of PJI and retrospective analysis of etiology in patients with PJI. Clin Chim Acta. 2020;506:67-71.

13. Hamer HM, Stroobants AK, Bavalia R, Ponjee G, Klok FA, van der Hulle T, Huisman MV, Hendriks HA, Middeldorp S. Diagnostic accuracy of four different D-dimer assays: a post-hoc analysis of the YEARS study. Thromb Res. 2021;201:18-22.

14. Morawietz L, Classen RA, Schröder JH, Dynybil C, Perka C, Skwara A, Neidel J, Gehrke T, Frommelt L, Hansen T, Otto M, Barden B, Aigner T, Stiehl P, Schubert T, Meyer-Scholten C, König A, Ströbel P, Rader CP, Kirschner S, Lintner F, Rüther W, Bos I, Hendrich C, Kriegsmann J, Krenn $\checkmark$. Proposal for a histopathological consensus classification of the periprosthetic interface membrane. J Clin Pathol. 2006;59(6):591-7.

15. Youden WJ. Index for rating diagnostic tests. Cancer. 1950;3(1):32-5.

16. Zhang Q, Dong J, Zhou D, Liu F. Circulating D-dimer versus Fibrinogen in the diagnosis of periprosthetic joint infection: a meta-analysis. Surg Infect (Larchmt). 2021;22(2):200-10.

17. Wang Y, Man Z, Yuan T, Cao H, Sun S. Reliability of D-dimer determination in diagnosis of peri-prosthetic joint infection. A systematic review and meta- analysis. Surg Infect (Larchmt). 2021;22(4):374-82.

18. Lu G, Li T, Ye H, Liu S, Zhang P, Wang W. D-dimer in the diagnosis of periprosthetic joint infection: a systematic review and meta-analysis. J Orthop Surg Res. 2020;15(1):265.

19. Yan J, Xie K, Jiang X, Han X, Wang L, Yan M. D-dimer for diagnosis of periprosthetic joint infection: a meta-analysis. J Orthop Sci. 2020;S0949-2658(20):30284.

20. Chen X, Li H, Zhu S, Wang Y, Qian W. Is D-dimer a reliable biomarker compared to ESR and CRP in the diagnosis of periprosthetic joint infection? A systematic review and meta-analysis. Bone Joint Res. 2020;9(10):701-8.

21. Zhang H, Sun $X$, Xin $P$, Zhu X, Jie K, Cao H, Feng W, Zeng Y, Lv Y, Chen J, Li J, Zeng J, Zeng Y. Diagnostic accuracy of D-dimer in periprosthetic joint infection: a diagnostic meta-analysis. J Orthop Surg Res. 2020;15(1):334.

22. Tian B, Cui $L$, Jiang $W$. The diagnostic effect of $a$-defensin, D-dimer, and IL-6 in periprosthetic joint infection: a systematic review and diagnostic meta-analysis. J Orthop Surg (Hong Kong). 2020;28(3):2309499020971861.

23. Pan L, Wu H, Liu H, Yang X, Meng Z, Cao Y. Fibrinogen performs better than D-dimer for the diagnosis of periprosthetic joint infection: a metaanalysis of diagnostic trials. J Orthop Surg Res. 2021;16(1):30.

24. Fu J, Ni M, Chai W, Li X, Hao L, Chen J. Synovial fluid viscosity test is promising for the diagnosis of periprosthetic joint infection. J Arthroplasty. 2019;34(6):1197-200.

25. Li R, Shao HY, Hao LB, Yu BZ, Qu PF, Zhou YX, et al. Plasma fibrinogen exhibits better performance than plasma D-dimer in the diagnosis of periprosthetic joint infection: a multicenter retrospective study. J Bone Joint Surg Am. 2019;101(7):613-9.

26. Xu H, Xie J, Huang Q, Lei Y, Zhang S, Pei F. Plasma fibrin degradation product and D-dimer are of limited value for diagnosing periprosthetic joint infection. J Arthroplasty. 2019;34(10):2454-60.

27. Wu H, Meng Z, Pan L, Liu H, Yang X, Yongping C. Plasma fibrinogen performs better than plasma d-dimer and fibrin degradation product in the diagnosis of periprosthetic joint infection and determination of reimplantation timing. J Arthroplasty. 2020;35(8):2230-6.

28. Ackmann T, Möllenbeck B, Gosheger G, Schwarze J, Schmidt-Braekling T, Schneider KN, Frommer A, Dieckmann R, Theil C. Comparing the 
diagnostic value of serum D-dimer to CRP and IL-6 in the diagnosis of chronic prosthetic joint infection. J Clin Med. 2020;9(9):2917.

29. Huang JC, Chen X, Qiang S, Zheng WD, Zheng J, Jin Y. Exciting performance of plasma fibrinogen in periprosthetic joint infection diagnosis. Orthop Surg. 2021. https://doi.org/10.1111/os.12964.

30. Chen X, Qian W, Weng X, Lin J, Wang Y, Zhu S. Different diagnostic performance of fibrinogen and $D$-dimer in periprosthteic joint infection: a propensity score matched study. BMC Musculoskelet Disord. 2021;22:422.

31. Pieper CT, Rao KM, Currie MS, Harris TB, Cohen HJ. Age, functional status and racial differences in plasma D-dimer levels in community dwelling eldery persons. J Gerontol A Biol Sci Med Sci. 2000;55:M649.

32. Grzelecki D, Walczak P, Grajek A, Szostek M, Dudek P, Bartosz P, Olewnik Ł, Czubak-Wrzosek M, Marczak D, TyraKowski M. Elevated plasma D-dimer concentration has higher efficacy for the diagnosis of periprosthetic joint infection of the knee than of the hip-A single-center, retrospective study. J Orthop Res. 2021;39(2):291-8.

33. Korte W, Riesen W. Latex-enhanced immunoturbidimetry allows D-dimer determination in plasma and serum samples. Clin Chem. 2000;46:871-2.

34. Li C, Margaryan D, Ojeda-Thies C, Carsten Perka C, Trampuz A. Meta-analysis of serum and/or plasma D-dimer in the diagnosis of periprosthetic joint infection. J Orthop Surg Res. 2020;15:298.

35. Lee YS, Lee YK, Han SB, Nam CH, Parvizi J, Koo KH. Natural progress of Ddimer following total joint arthroplasty: a baseline for the diagnosis of the early postoperative infection. J Orthop Surg Res. 2018;13:36.

\section{Publisher's Note}

Springer Nature remains neutral with regard to jurisdictional claims in published maps and institutional affiliations.

- fast, convenient online submission

- thorough peer review by experienced researchers in your field

- rapid publication on acceptance

- support for research data, including large and complex data types

- gold Open Access which fosters wider collaboration and increased citations

- maximum visibility for your research: over $100 \mathrm{M}$ website views per year

At BMC, research is always in progress.

Learn more biomedcentral.com/submissions 